\title{
Transthoracic contrast echocardiography for detection of pulmonary arteriovenous malformations in hereditary hemorrhagic telangiectasia: How can the process be improved for patient safety?
}

\author{
Xiangning Fan ${ }^{1}$, J onathan Choy ${ }^{1,2}$, Miriam Shanks ${ }^{1,2}$, Dilini P. Vethanayagam ${ }^{1,3}$ \\ 1. Department of Medicine, University of Alberta, Edmonton, AB, Canada. 2. Mazankowski Heart Institute, Edmonton, AB, \\ Canada. 3. Edmonton HHT Center, Edmonton, AB, Canada.
}

Correspondence: Dilini P. Vethanayagam. Address: 3-105 Clinical Sciences Building, University of Alberta, Edmonton, AB, T6G 2G3, Canada. Email: dilini@ualberta.ca

Received: January 28, $2014 \quad$ Accepted: March 2, $2014 \quad$ Online Published: March 17, 2014

DOI : 10.5430/ijdi.v1n2p18 URL: http://dx.doi.org/10.5430/ijdi.v1n2p18

\section{Abstract}

Introduction: Patients with hereditary hemorrhagic telangiectasia (HHT) are at risk for pulmonary arteriovenous malformations (PAVMs). Extracardiac right-to-left shunts due to PAVMs can lead to systemic emboli; clinical sequelae including strokes and brain abscesses. Regular screening is required, with appropriate medical/interventional management to alleviate morbidity and mortality from PAVMs. International HHT guidelines recommend standardized transthoracic contrast echocardiogram (TTCE) with agitated saline (in an HHT Foundation-accredited laboratory) as the initial screening test, and, when needed, non-contrast computerized tomography of the chest (CT Chest) to follow up on a positive TTCE.

Case presentation: A 40-year-old woman was seen for evaluation of HHT and referred for TTCE as part of her PAVM screen. Following administration of agitated saline, she developed a headache, visual changes in her right eye, and right arm weakness, followed by posturing and stiffness in her right arm. Her symptoms resolved after five minutes, consistent with a transient ischemic attack (TIA).

Clinical impact: Protocols at our institution were amended following this index case and echocardiography laboratory protocols were adjusted to use a maximum of $0.1 \mathrm{cc}$ air to $9.9 \mathrm{cc}$ saline for TTCE studies in the HHT population. Only the attending echocardiologist who is familiar with possible systemic air embolism during bubble contrast echocardiography in patients with HHT should perform agitation and infusion of contrast and should directly review the images for appropriate visualization during the exam for this test to be appropriately interpreted. Paradoxical emboli due to right-to-left shunt are important to recognize. Best practice for PAVM screening in HHT patients includes use of standardized protocols for the performance and reporting of TTCE in the evaluation of pulmonary shunts.

\section{Keywords}

Hereditary hemorrhagic telangiectasia, Osler-Weber-Rendu syndrome, Pulmonary arteriovenous malformations, Contrast echocardiography, Pulmonary screening 


\section{I ntroduction}

Hereditary hemorrhagic telangiectasia (HHT) is an autosomal dominant genetic disorder of angiogenesis estimated to affect 1 in 5000 people ${ }^{[1]}$. Clinically the diagnosis can be established definitively by the presence of at least three of the following: (a) recurrent epistaxis, (b) mucocutaneous telangiectasias, (c) visceral arteriovenous malformations, and/or (d) confirmed HHT in a first degree relative ${ }^{[1]}$. The diagnosis can also be made through molecular genetic testing in an individual who has a first degree relative with HHT and a known familial gene mutation ${ }^{[1]}$.

Pulmonary arteriovenous malformations (PAVMs) exist in 15\%-50\% of individuals with $\mathrm{HHT}^{[1]}$, resulting in an extracardiac right-to-left shunt directly connecting the venous and the systemic arterial circulations. PAVMs may have catastrophic consequences that can be averted with routine pre-symptomatic screening and preventative transcatheter embolotherapy when found. We present a patient with HHT who developed symptoms suggesting a transient ischemic attack (TIA) from paradoxical air embolism following agitated saline injection during transthoracic contrast echocardiography (TTCE) to screen for PAVMs. The discussion following the case will review complications of PAVMs in HHT, and discuss the role of screening for PAVMs with TTCE in this genetic disorder.

\section{Case presentation}

A 40 year old woman with definite HHT was referred for multi-system screening for visceral arteriovenous malformations (including PAVMs) ${ }^{[1]}$. She had a history of recurrent epistaxis, but was otherwise healthy.

Screening for PAVMs was performed with TTCE, using agitated saline as a contrast agent. During TTCE, three intravenous agitated saline injections, each containing approximately $0.5 \mathrm{cc}$ of air, were performed within a five minute interval. Approximately one minute after the last injection, the patient developed symptoms of flashing lights in her right eye and flaccid right arm weakness, followed by posturing and stiffness in her right arm. Additionally, she described a bilateral occipital headache. Her symptoms lasted 5 minutes, then completely resolved (see Figure 1).

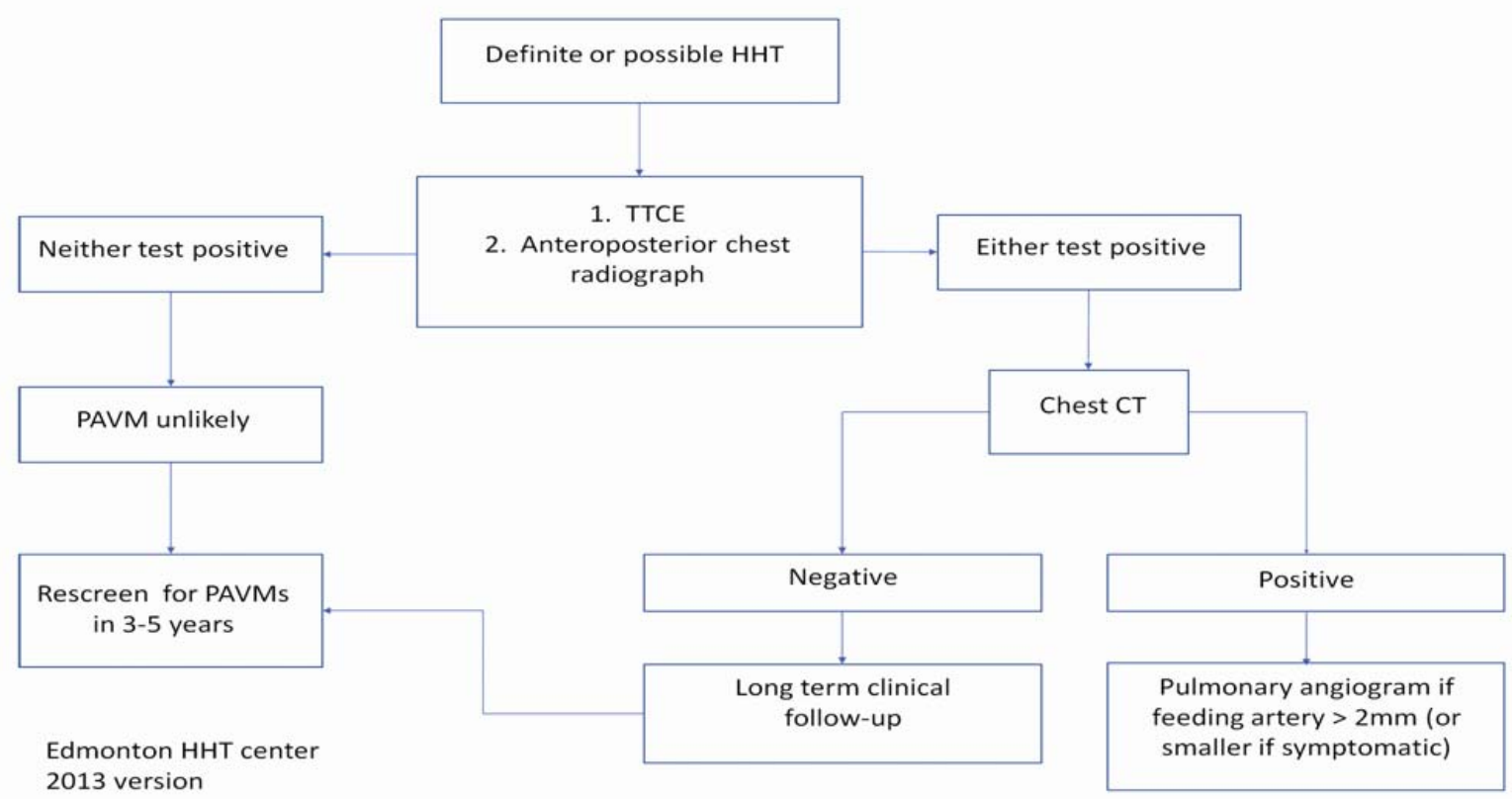

Figure 1. Edmonton screening algorithm for PAVMs in patients with HHT 
Cerebral computerized tomography (CT) and magnetic resonance imaging immediately following this event did not reveal any evidence of a cerebral vascular accident, and her symptoms were felt to be most consistent with a TIA.

Her echocardiogram revealed air bubbles in the left atrium after approximately 3 seconds. Direct visualization of the inter-atrial septum during injection of agitated saline ruled out an atrial septal defect causing an intra-cardiac shunt. The late appearance of bubbles in the left atrium suggested an extra-cardiac nature to the noted right-to-left shunt, related in the HHT population to PAVMs. Subsequently a CT scan of the chest revealed a solitary PAVM with a single 2 mm feeding artery and draining vein. She was offered embolization of her PAVM, but declined in favour of clinical and radiographic monitoring to determine the rate of growth of her PAVM over time, given the small size of the feeding artery. She was educated on precautions suggested to individuals with PAVMs to reduce the risk of paradoxical embolism (use of air-eliminating intravenous filters for venous access and Vacutainer ${ }^{\circledR}$-type devices for blood draws) and cerebral abscess (antibiotics with dental cleaning and other procedures associated with intermittent bacteremia).

\section{Discussion}

Observational studies have established that between $15 \%-50 \%$ of patients with HHT have PAVMs ${ }^{[1]}$. Major complications of PAVMs in patients with HHT include both embolic (e.g. stroke secondary to paradoxical embolism and brain abscesses secondary to transient bacteremia seeding the brain) and hemorrhagic (e.g. pulmonary hemorrhage) events. Neurologic complications (e.g. cerebral abscess, ischemic stroke and TIA) have been described to occur in approximately $30 \%$ of all patients with PAVMs ${ }^{[2]}$, most of whom also have HHT. Additionally, a relationship between right to left shunts and migraine with aura, symptoms of which may mimic acute stroke, has been previously described in the HHT population in the literature ${ }^{[3]}$. Among patients with multiple PAVMs, the risk of neurologic complications appears to be increased further ${ }^{[4]}$. However, early detection and embolization of PAVMs can reduce neurologic complications in individuals with $\mathrm{HHT}^{[1,5]}$.

Chest radiography, arterial blood gas measurement with alveolar-arterial gradient assessment when the patient is breathing $100 \%$ inspired oxygen, TTCE, and radionucleotide perfusion scanning have all been used to screen for PAVMs ${ }^{[4-7]}$. Conventional pulmonary angiography or chest CT is considered the gold standard for detection of PAVMs ${ }^{[1]}$. Among screening modalities, TTCE appears to be the single most sensitive screening test for PAVMs ${ }^{[5]}$. TTCE alone ${ }^{[8]}$ or in combination with plain chest radiography ${ }^{[5]}$ has been reported to be $100 \%$ sensitive for, and able to definitively rule out, PAVMs in patients with shunts. At least some authors have argued, however, that addition of plain chest radiography to TTCE exposes patients to ionizing radiation without demonstrated improvement in detection of PAVMs ${ }^{[9]}$. Given the need for lifelong screening required in HHT, minimizing use of modalities which utilize ionizing radiation is preferred to reduce the cumulative lifetime radiation exposure ${ }^{[1,5]}$.

Importantly, in a recent case series of individuals with HHT and PAVMs, only a minority of patients (29\%) were diagnosed with PAVMs through systematic screening, with other cases revealed as a result of incidental imaging findings or after development of complications ${ }^{[2]}$. This suggests that greater awareness is needed, to allow for pre-symptomatic screening of HHT patients for PAVMs.

Agitated saline is the preferred contrast agent for TTCE in individuals with HHT ${ }^{[1]}$. Air bubbles are normally unstable in the normal pulmonary circulation and are removed within the alveolar capillary bed prior to entering the pulmonary venous circulation. However, up to $20 \%$ of normal, healthy individuals may have an intrapulmonary shunt at rest ${ }^{[10]}$. The clinical significance of a small degree of shunting in patients without HHT is unclear.

Use of agitated saline only within experienced hands during echocardiography is vital to minimize the risk of procedure-related paradoxical emboli, as cerebral ischemic events are a known but rare complication of TTCE with agitated saline if intra-cardiac or extra-cardiac shunts exist ${ }^{[11]}$. Air to saline ratios of $1 \mathrm{cc}$ air to 9 cc saline have been 
reported in both the HHT ${ }^{[5]}$ and non-HHT ${ }^{[12]}$ echocardiography literature, and are widely used in community echocardiography laboratories.

Due to the presumed paradoxical air embolism this patient experienced during TTCE using agitated saline containing 0.5 cc air, the current protocol at our institution was modified such that at most only 0.1 cc of air in 9.9 cc of saline is used during TTCE in patients with HHT and suspected shunt. It is only if the initial injection is negative or equivocal that a repeat injection would be considered. To optimize patient safety, diagnostic testing is performed only with the echocardiologist-who is aware of the higher potential for embolic complications-present during the procedure, and with an echocardiologist who frequently performs TTCE. Improved visual discrimination (when needed) is provided by back-aspiration of a small amount $(0.1 \mathrm{cc}-0.2 \mathrm{cc})$ of the patient's own blood, and mixing this with the agitated saline ${ }^{[13]}$; this practice could contribute to the safety of TTCE in patients with right-to-left shunts by decreasing the amount of air required for good visualization, and, thus, decreasing the risk of air embolization. The echocardiologist directly instills the contrast, and reviews real-time images to ensure appropriate visualization during the exam. Center accreditation by the HHT Foundation International (including an echocardiography laboratory process review) is ideal (as with our laboratory) to ensure echocardiography laboratory methods are consistent with international standards.

\section{Conclusion}

Standardized TTCE is the modality of choice to screen patients with HHT for PAVMs, especially in HHT centers with the requisite expertise in performing this screening. Recent literature ${ }^{[9]}$ has highlighted the possibility that TTCE alone may be sufficient for screening purposes, although this remains controversial.

Risk of air embolism during contrast echocardiography can be minimized through standardizing the performance and interpretation of the procedure and utilizing an echocardiography laboratory that has sufficient volume to have the requisite expertise in performance and interpretation.

We suggest PAVM screening in the HHT population only with standardized TTCE, ideally performed by echocardiologists who are experienced to perform this test in patients with right-to-left shunt, and are knowledgeable of the nuances in technique and procedures required for safe and effective screening diagnostics for this population.

\section{References}

[1] Faughnan, ME, Palda VA, Garcia-Tsao G, Geisthoff UW, McDonald J, Proctor DD, et al. International Guidelines for the Diagnosis and Management of Hereditary Hemorrhagic Telangiectasia. J Med Genet. 2011; 48: 73-87. http://dx.doi.org/10.1136/jmg.2009.069013

[2] Cottin V, Chinet T, Lavole A, Corre R, Marchand E, Reynaud-Gaubert M, et al. Pulmonary Arteriovenous Malformations in Hereditary Hemorrhagic Telangiectasia. Medicine. 2007; 86: 1-17. http://dx.doi.org/10.1097/MD.0b013e31802f8da1

[3] Post MC, van Gent MWF, Plokker HWM, Westermann CJJ, Kelder JC, Mager JJ, et al. Pulmonary arteriovenous malformations associated with migraine with aura. Eur Resp J. 2009; 34: 882-7. http://dx.doi.org/10.1183/09031936.00179008

[4] Moussouttas M, Fayad P, Rosenblatt M, Hashimoto M, Pollak J, Henderson K, Ma TY-Z, White RI. Pulmonary arteriovenous malformations: Cerebral ischemia and neurologic manifestations. Neurology. 2000; 55: 959-64. http://dx.doi.org/10.1212/WNL.55.7.959

[5] Cottin V, Plauchu H, Bayle J-Y, Barthelet M, Revel D, Cordier J-F. Pulmonary Arteriovenous Malformations in Patients with Hereditary Hemorrhagic Telangiectasia. Am J Respir Crit Care Med. 2004; 169: 994-1000. http://dx.doi.org/10.1164/rccm.200310-1441OC

[6] Lee WL, Graham AF, Pugash RA, Hutchison SJ, Grande P, Hyland RH, et al. Contrast Echocardiography Remains Positive After Treatment of Pulmonary Arteriovenous Malformations. Chest. 2003; 123: 351-8. http://dx.doi.org/10.1378/chest.123.2.351 
[7] Barzilai B, Waggoner AD, Spessert C, Picus D, Goodenberger D. Two-dimensional contrast echocardiography in the detection and follow-up of congenital pulmonary arteriovenous malformations. Am J Cardiology. 1991; 68: 1507-10. http://dx.doi.org/10.1016/0002-9149(91)90287-U

[8] Gazzaniga P, Buscarini E, Leandro G, Reduzzi L, Grosso M, Pongiglione G, Pedrinazzi C, et al. Contrast echocardiography for pulmonary arteriovenous malformations screening: does any bubble matter? Eur J Echocardiogr. 2009; 10: 513-18. http://dx.doi.org/10.1093/ejechocard/jen317

[9] Van Gent MW, Post MC, Luermans JG, Snijder RJ, Westermann CJ, Plokker HW, et al. Screening for pulmonary arteriovenous malformations using transthoracic contrast echocardiography: a prospective study. Eur Respir J. 2009 Jan; 33(1): 85-91. http://dx.doi.org/10.1183/09031936.00049008

[10] Lovering AT and Goodman RD. Detection of Intracardiac and Intrapulmonary Shunts at Rest and During Exercise Using Saline Contrast Echocardiography. In: Ainslie P, editor. Applied Aspects of Ultrasonography in Humans. Rijeka, Croatia: InTech Europe; 2012. 159-174 p.

[11] Romero JR, Frey JL, Schwamm LH, Demaerschalk BM, Chaliki HP, Parikh G, et al. Cerebral Ischemic Events Associated With 'Bubble Study' for Identification of Right to Left Shunts. Stroke. 2009; 40: 2343-48. http://dx.doi.org/10.1161/STROKEAHA.109.549683

[12] Attaran RR, Ata I, Kudithipudi V, Foster L, Sorrell VL. Protocol for Optimal Detection and Exclusion of a Patent Foramen Ovale Using Transthoracic Echocardiography with Agitated Saline Microbubbles. Echocardiography. 2006; 23: 616-22. http://dx.doi.org/10.1111/j.1540-8175.2006.00272.x

[13] Fan S, Nagai T, Luo H, Atar S, Naqvi T, Birnbaum Y, et al. Superiority of the Combination of Blood and Agitated Saline for Routine Contrast Enhancement. J Am Soc Echocardiogr. 1999; 12: 94-98. http://dx.doi.org/10.1016/S0894-7317(99)70120-3 